\title{
Long Period Grating in Multicore Optical Fiber: an Ultra-Sensitive Vector Bending Sensor for Low Curvatures
}

\author{
Pouneh Saffari, ${ }^{1}$ Thomas Allsop, ${ }^{2}$ Adedotum Adebayo, ${ }^{2}$ David Webb, ${ }^{2}$ Roger Haynes, ${ }^{1}$ \\ Martin M. Roth ${ }^{1}$ \\ ${ }^{I}$ Leibniz-Institut für Astrophysik Potsdam (AIP), An der Sternwarte 16, D-14482 Potsdam, Germany \\ ${ }^{2}$ Aston Institute of Photonic Technologies, Aston University, Aston Triangle, Birmingham, B4 7ET, UK
}

\begin{abstract}
Long period grating were UV inscribed into a multi-core fiber consisting of 120 single mode cores. The multi-core fiber that hosts the grating was fusion spliced into single mode fiber at both ends. The splice creates a taper transition between the two types of fiber that produces a non-adiabatic mode evolution; this results in the illumination of all the modes in the multi-core fiber. The spectral characteristics of this fiber device as a function of curvature were investigated. The device yielded a significant spectral sensitivity as high as $1.23 \mathrm{~nm} / \mathrm{m}^{-1}$ and $3.57 \mathrm{~dB} / \mathrm{m}^{-1}$ to the ultra-low curvature values from 0 to $1 \mathrm{~m}^{-1}$. This fiber device can also distinguish the orientation of curvature experienced by the fiber by the long period grating attenuation bands producing either a blue or red wavelength shift. Finite Element Method model was used to investigate the modal behaviour in multi-core fiber and to predict the phase-matching curves of the long period grating inscribed into multi-core fiber.
\end{abstract}

Long Period Grating (LPG) is a large scale $(10 \mu \mathrm{m}$ to $1 \mathrm{~mm}$ ) periodic perturbation of the refractive index of the core of a photosensitive single mode optical fiber [1]. The conventional LPG couples the core mode to various forward propagating cladding modes that fulfill the phase-matching condition of the grating. Since the cladding modes suffer from high attenuation as they are mainly absorbed by the coating layer of the fiber or scattered out, the transmission spectrum of LPG consists of a series of attenuation bands [1]. LPGs have shown potentials in the field of telecommunications [2] and are a topic of research in environmental sensing through their sensitivity to strain, bending, temperature, and the refractive index of surrounding medium [3]. The LPG's response to the mentioned stimuli can manifest itself in two ways: first the spectral shift of its associated attenuation band (wavelength shift of stop-bands) and second the change in the spectral transmission profile of the attenuation band. Comparing to the traditional Fiber Bragg Gratings (FBGs), that are more sensitive to the axial strain and almost insensitive to the curvature measurements, LPGs are very sensitive to the applied bending. However, the bending sensitivity of conventional uniform LPGs, which are written in standard step index single mode fibers [3], are independent of the bending orientation. On the other hand, the ability to determine the direction of the deformation can be very important for sensors used in structural health monitoring applications including monitoring the condition of bridges, cranes, blades of wind turbines, etc. Therefore there is a high demand for developing sensors with the ability of measuring both direction of bend and its amplitude. There are many successful examples in the literature of employing standard LPGs in various radially asymmetric fibers [4-8] or inscribed asymmetric gratings in radially symmetric fiber [9-16] to create a directional bend sensor. However, none of the introduced sensors shows a high sensitivity to small curvatures and strong directional sensitivity. Furthermore, complexity and expensive fabrication approach, as well as the difficulty of splicing some of these fibers to the ordinary fibers, are considered as significant drawbacks of these approaches. In this paper, for the first time to our knowledge, we present a UV inscribed LPG written into a Multi-Core Fiber (MCF), which is highly sensitive to bending at low curvatures and can also sense the orientation of the curvature. Comparing with the LPGs written in standard step index fibers no splitting effect, originating from the induced birefringence [17], was observed from this device. A Finite Element Method (FEM) was used to investigate the modal behavior of $\mathrm{MCF}$, spectral characteristics of the LPG device, and to predict the phase matching curve and fabrication design parameters of the LPG device.

FEM modeling (Comsol software) was used to investigate the possible core and cladding modes that the MCF would support. A series of simulations were conducted over a range of wavelengths to obtain the effective indices of the core and cladding modes.

Fig. $1(\mathrm{a}-\mathrm{d})$ illustrate the two distinctive classes of modes: one where most of the light propagates in the cores, i.e. the "core modes" [presented in Fig. 1 (a, b)]; the other where the light propagates more uniformly over the whole core and cladding region i.e. the "cladding modes", [shown in Fig. 1 (c, d)]. FEM simulation generated a series of core and cladding modes. By visual inspection of the generated modes and using conventional waveguide theory [18], it was decided that modes with the radial symmetry of the electric field are the real solutions for FEM model. Effective index values of approximately 1.45 for the 
core and 1.44 to 1.4426 for the cladding modes were decided for the MCF and used to predict the possible phase matching condition between core modes, core to cladding modes, and cladding to cladding modes. Using the above premises, we obtained 110 core modes and investigated the coupling coefficients for all of the modes. It was found that only relatively few significant coupling between core modes occur and no core modes coupling meet the phase matching conditions in the wavelength range of interest.

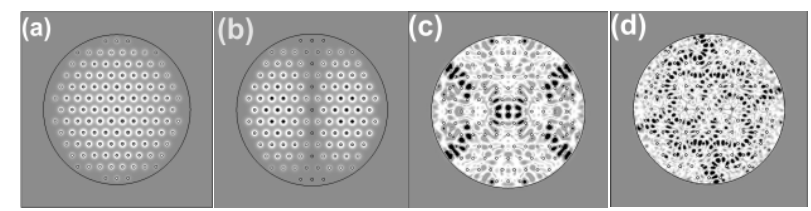

Fig. 1. Images of modes constructed from FEM modeling (a) core mode with effective index of 1.44709 (b) core mode with effective index of 1.44788 (c) cladding modes with effective index of 1.44261 (d) cladding modes effective index of 1.44003 at a wavelength of $1500 \mathrm{~nm}$.

The phase matching condition between the guided mode and the forward propagating cladding modes in LPG is given by:

$$
\beta_{01}-\beta_{c l}^{(n)}=\frac{2 \pi}{\Lambda}
$$

Where $\beta_{01}$ and $\beta_{c l}^{n}$ are the propagation constant of the core mode and cladding modes respectively. $\Lambda$ denotes the periodicity of the grating required to couple the fundamental mode to the $\mathrm{n}^{\text {th }}$ cladding mode. One can predict the wavelength at which the mode coupling occurs from the grating period [1]. FEM simulations were used to predict the phase matching curves that are presented in Fig. 2 (a) and we can choose the desired coupling wavelength region of the LPG by selecting the grating period from these curves. The MCF employed for the experiment consists of 120 Ge doped silica single mode cores. Each core has a diameter of $3.9 \mu \mathrm{m}$ and the distance between the adjacent cores is $16 \mu \mathrm{m}$. The fiber cladding is pure silica and has an outer diameter of $230 \mu \mathrm{m}$. A $10 \mathrm{~cm}$ long length of MCF was first fusion spliced, at both ends, to the SMF and then the unit was hydrogen loaded at 120 bar and room temperature for a period of two weeks. LPG was written into the MCF in a single exposure using a $\mathrm{CW}^{-} 244 \mathrm{~nm}$ frequency-doubled $\mathrm{Ar}^{+}$laser operating at $\sim 95 \mathrm{~mW}$. The spot diameter of the laser beam at the position of fiber was $\sim 120 \mu \mathrm{m}$ and only partially covered the cross section of cores within the MCF $(\sim 180 \mu \mathrm{m})$. For the LPG fabrication, we employed point-by-point inscription technique with the scan velocity of $0.10 \mathrm{~mm} / \mathrm{s}$. The respective length and period of LPG were designed to be $50 \mathrm{~mm}$ and $325 \mu \mathrm{m}$. The LPG period was specifically selected for mode coupling at $1500 \mathrm{~nm}$ region. The attenuation band of the LPG was characterized by employing an Optical Spectrum Analyzer (OSA) with a resolution of $0.2 \mathrm{~nm}$ and a BroadBand light Source (BBS). Fig. 2 (b) shows the transmission spectrum of the fabricated device.
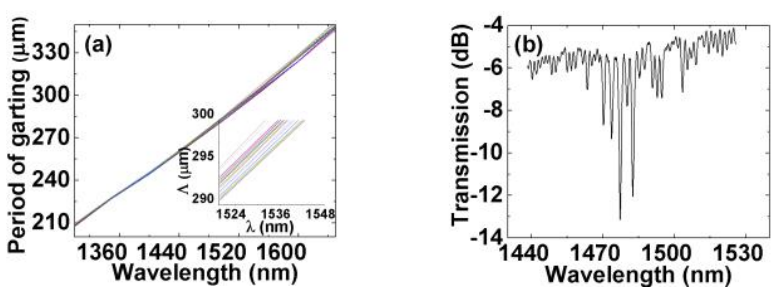

Fig. 2. (a) Phase matching curve for LPG written in MCF using core mode (0) over the wavelength range of interest (b) Experimental transmission profile of LPG written in MCF.

The fusion splicing between the SMF and MCF was visually inspected and the physical dimensions from SMF to MCF was measured using a microscope system (Axioskop, Zeiss); it was found that the transition between the two fibers produced a taper (Fig. 3(a)). The fiber taper effect on the evolution of the cladding modes and the core modes (adiabatic or non-adiabatic) across the taper length was investigated using the slowness criterion [19-22]. This is done by considering the magnitude of the rate of change of the radius as a function of the beat length between the modes being investigated. These values were compared to the ratio of taper's radius to beat lengths of the modes along the taper length that is shown in Fig. 3 (b). Slowness criterion for adiabatic mode evolution is given by [18]:

$$
\left|\frac{\partial r}{\partial z_{b}}\right|<<\frac{r}{z_{b}}, z_{b}=\frac{2 \pi}{\beta_{1}-\beta_{2}}
$$

Where $z_{b}$ is the beat length between two modes with propagation constant of $\beta_{1}$ and $\beta_{2}, r$ is the radius of the fiber along the taper and $r / z_{b}$ is the adiabatic length scale criteria. The modes chosen for analysis were selected from the phase matching curve shown in Fig. 2 (a) and the satisfied matching condition around the spectral location of $1500 \mathrm{~nm}$. Three limit curves shown in Fig. 3 (b) correspond to the particular mode propagation constants with the larges beat lengths that were calculated along the taper length using FEM simulation (Comsol) for the investigated wavelengths: $1400 \mathrm{~nm}$ (dotted line), $1500 \mathrm{~nm}$ (dashed line) and,1650 $\mathrm{nm}$ (solid line). The section lengths of the taper for the simulation were chosen to ensure that the E-fields do not significantly change their phase along that section compared to the light guided in a non-tapered fiber of the same initial diameter [21].

Tapering caused an overall reduction in the radius of the MCF from $115 \mathrm{~mm}$ to $62.5 \mathrm{~mm}$. Hence, it was assumed that there was a reduction in the radius of the cores from $4 \mu \mathrm{m}$ to $2.1 \mu \mathrm{m}$ and core separation from 16 $\mu \mathrm{m}$ to $7.8 \mu \mathrm{m}$. Therefore, there will be 7 cores from $\mathrm{MCF}$ with direct coupling to the SMF core. Also each core in the MCF yields a V parameter of $\sim 1.44$; this results in approximately $52 \%$ of the intensity distribution extending out of the cores with an effective radius of $\sim 5 \mu \mathrm{m}$ carrying the major part of the energy. This implies a significant coupling between cores and 
the cladding especially when the slowness criteria is investigated and the beat length between the core and cladding modes ranges upward from $10 \mu \mathrm{m}$. As shown in Fig. 3 (a), the taper length is approximately $50 \mu \mathrm{m}$ thus the beat length is short compared to the length of the taper and significant coupling occurs across the cores. Inspecting Fig. 3 (b), section B of the taper exhibits the condition of non-adiabatic mode evolution; therefore strong coupling occurs between the core modes to the cladding modes and the cladding to the cladding modes whilst sections $\mathrm{A}$ and $\mathrm{C}$ have adiabatic condition that implies a negligible power transfer between the core and the cladding modes. Overall the section B explains the observed low-loss fiber device (measured for several prepared samples $\sim 2 \mathrm{~dB}$ ) created by the union of these two fibers.
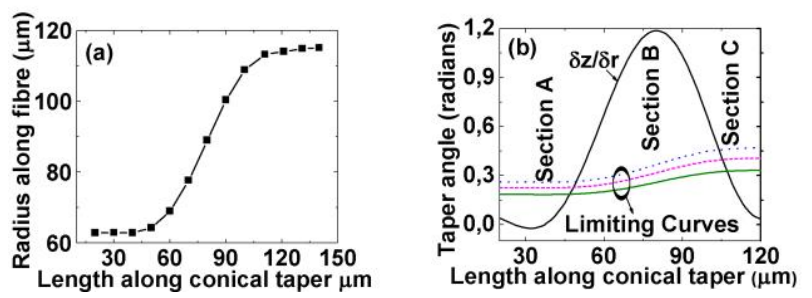

Fig. 3 (a). Typical measured geometric profile of a conical taper/splice between the SMF and MCF (b) The ratio of taper's radius to beat lengths of the modes along the taper length (three slowness criterion- limit curves for the largest beat lengths calculated for three wavelengths: $1400 \mathrm{~nm}$ (dotted line), $1500 \mathrm{~nm}$ (dashed line) and, $1650 \mathrm{~nm}$ (solid line)).

Four fiber devices were fabricated and all subjected to the bending measurements. For this purpose, devices were clamped mid-way between two towers on a flexible V-groove; both clamps were mounted on translation stages, which were moved inwards to induce a bend in the groove and hence the attached device. It should be noted that in order to prevent the strain cross sensitivity [23] for this set of measurements, the samples were attached to the groove from one end while its other end was free. Furthermore, rotation of the LPG sensors was performed on this rig by subjecting the sensors to a series of known curvatures while rotating the sensor around its clamp. The tags on the fibers were used to ensure that there was no twist in the fibers during the experiments and also to indicate the orientation of the fibers. The experimental setup for bending measurements is presented in Fig. 4. The light from the BBS was passed through a broadband polarizer before illuminating the LPG device. The device's curvature is calculated by [6]:

$$
R=\frac{2 d}{\left(d^{2}+L^{2}\right)}
$$

where $\mathrm{L}$ is the half distance between the edges of the two towers and $d$ is the deflection at the center of the LPG.

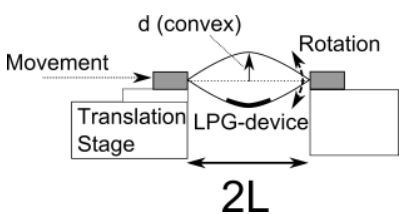

Fig. 4. Schematic diagram of the experimental setup for bending measurements.

The devices were subjected to a range of curvatures from 0 to $1 \mathrm{~m}^{-1}$ and the transmission spectra were collected using an OSA with the resolution of $0.2 \mathrm{~nm}$. These measurements were repeated for two opposite bending directions, convex and concave. A typical experimental results for a single fiber device are shown in Fig. 5 ( $\mathrm{a}$ and b). Inspecting Fig. 5 ( $\mathrm{a}$ and $\mathrm{b}$ ), the LPG attenuation bands exhibit either blue or red wavelength shift depending on the direction of the applied curvature while their optical strength reduce with the increase in curvature value.
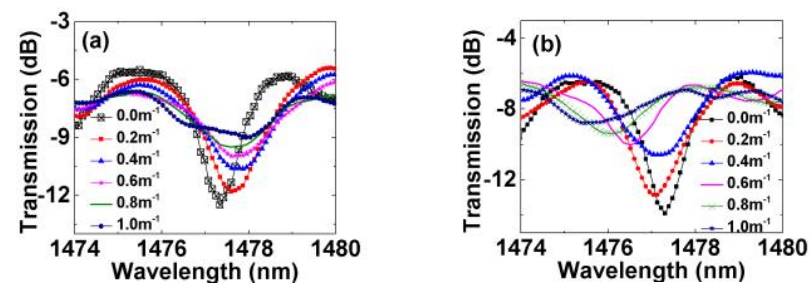

Fig. 5. The transmission spectra of the proposed sensor while it was subjected to a range of (a) convex and (b) concave bending curvature from 0 to $1 \mathrm{~m}^{-1}$.

Fig. 6 (a) shows the spectral sensitivity of the sensor when subjected to a range of curvatures in both of the convex and concave directions. The device exhibits different linear spectral responses to the convex and concave curvatures as a result of the asymmetric inscription of the LPG structure. This leads to the respective blue and red shift in the central wavelength of its stop-bands, as seen in Fig. 6 (a). As previously mentioned, the full cross section of the MCF core region, $\sim 180 \mu \mathrm{m}$, is significantly larger than the inscription beam diameter $(\sim 120 \mu \mathrm{m})$ resulting in LPG inscription in only 46 cores of the MCF. Hence the LPG was written across an asymmetric cross section of the MCF cores. This device also shows a linear response to both concave and convex curvatures with the change in its optical strength shown in Fig. 6 (b). The device shows the respective sensitivity of 3.57 $\mathrm{dB} / \mathrm{m}^{-1}$ and $3.15 \mathrm{~dB} / \mathrm{m}^{-1}$ for the concave and convex curvatures. The device yielded a bending sensitivity of $+0.49 \mathrm{~nm} / \mathrm{m}^{-1}$ and $1.23 \mathrm{~nm} / \mathrm{m}^{-1}$ to a low range value (up to $1 \mathrm{~m}^{-1}$ ) of convex and concave curvatures respectively. A theoretical investigation into the spectral sensitivity as a function of curvature was performed using Comsol (FEM software package) and conformal mapping technique and found a reasonable agreement with experimental investigation. The spectral sensitivities of this sensor are significantly greater in the lower curvature regime than any other researchers' published results. 


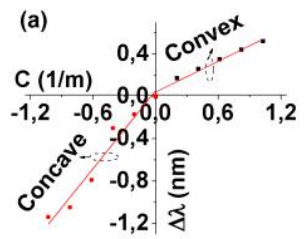

ig. 6. Response of the proposed sensor to convex and, concave curvatures in form of (a) wavelength shift (b) optical strength variation of LPG peak.

The spectral behavior of the sensors in the literature $[6,7,10,11,14-16]$ show limited sensitivities in the low curvature regime, but there are only a few number of data points resulting in some uncertainty in the sensitivity measurement in this range [12, 13]. Fig. 6 (a and b) show a difference in the magnitude of the device's sensitivity to the applied convex and concave curvatures. Considering the large diameter of the MCF, when it is subjected to a bend, there is a rather noticeable difference between the amount of curvature which is experienced by the inner and the outer bend. The asymmetric structure of the device and also the inscription orientation of the LPG (realized from the attached tags on the fiber) suggest that for the concave measurements the LPG was mainly located at the inner bend while over the convex measurements it was situated mostly at the outer bend. As a result, the spectral sensitivity of LPG, for which the wavelengths shift of the central LPG peak is measured, for the convex curvature is almost half of the correspondent measured value for the concave bending. This suggests that the LPG was experiencing a larger curvature during the concave bending measurements. This argument is further supported by the rate of the optical intensity variations of the LPG with the applied convex and concave curvatures; in this case, the bending sensitivity of the device for convex measurements is smaller than the concave measurements indicating that less curvature being experienced by LPG over the convex measurement.

In this paper, we have introduced a multi-core fiber based LPG bending sensor with an ultra-high sensitivity to the applied low curvature values and capability of detecting the bending orientation. The fabrication of the LPG was by UV inscription into an MCF containing 120 photosensitive single mode cores, for the first time to our knowledge. The detailed theoretical investigation of mode excitation in MCF is studied for an MCF that has been fusion spliced to the SMF-28 at both ends; also the splicing condition and mode evolution in the tapered region has been discussed. This fiber sensor has the compelling benefit of a simple and robust structure, capability of detection of small deflections with a high resolution and indicating the direction of the applied bend simultaneously.

Authors acknowledge the University of Bath for providing the MCF.

\section{References}

[1] S. Vasilev, and O. Medvedkov, Proc. SPIE 4083, (2000).
[2] K. O. Hill, B. Malo, K. Vineberg, F. Bilodeau, D. Johnson, and I. Skinner, Electron. Lett. 26, 1270 (1990). [3] V. Bhatia and A. M. Vengsarkar, Opt. Lett. 21, 692 (1996).

[4] H. J. Patrick, Electron. Lett. 36, 1763 (2000).

[5] X. F. Chen, C. Zhang, D. J. Webb, K. Kalli, and G. D. Peng, "Highly sensitive bend sensor based on Bragg grating in eccentric core polymer fiber," IEEE Photon. Technol. Lett. 22, 850 (2010).

[6] T. Allsop, A. Gillooly, V. Mezentsev, T. EarthgrowlGould, R. Neal, D. J. Webb, and I. Bennion, IEEE Trans. Instrum.Meas. 53, 130 (2004).

[7] D. H. Zhao, X. F. Chen, K. M. Zhou, L. Zhang, I. Bennion, W. N. MacPherson, J. S. Barton, and J. D. C. Jones, Appl. Opt. 43, 5425 (2004).

[8] F. M. Araujo, L. A. Ferreira, J. L. Santos, and F. Farahi, Meas. Sci. Technol. 12, 829 (2001).

[9] Y. J. Rao, Y. P. Wang, Z. L. Ran, and T. Zhu, J. Lightwave Technol. 21, 1320 (2003).

[10] T. Allsop, K. Kalli, K. Zhou, Y. Lai, G. Smith, M. Dubov, D. J. Webb, and I. Bennion, Opt. Commun. 281, 5092 (2008).

[11] H. J. Patrick, C. C. Chang, and S. T. Vohra, Electron. Lett. 34, 1773 (1998).

[12] T. Allsop, M. Dubov, A. Martinez, F. Floreani, I. Khrushchev, D. J. Webb, and I. Bennion, J. Lightwave Technol. 24, 3147 (2006).

[13] T. Allsop, M. Dubov, A. Martinez, F. Floreani, I. Khrushchev, D. J. Webb, and I. Bennion, Electron. Lett. 41, 59 (2005).

[14] L. Jin, W. Jin, and J. Ju, J. Lightwave Technol. 27, 4884 (2009).

[15] L. Y. Shao, L. Y. Xiong, C. K. Chen, A. Laronche, and J. Albert, J. Lightwave Technol. 28, 2681 (2010).

[16] S. Zhang, W. G. Zhang, S. C. Gao, P. C. Geng, and X. L. Xue, Opt. Lett. 37, 4480 (2012).

[17] Y. Liu, L. Zhang, J. A. R. Williams, and I. Bennion, IEEE Photon. Technol. Lett. 12, 531 (2000).

[18] A. W. Synder and J. D. Love, Optical Waveguide Theory. London, U.K: Chapman and Hall, (1983).

[19] T. Allsop F. Floreani, K. Jedrzejewski, R. Marquez, R. Romero, D. J. Webb, and I. Bennion, Electron. Lett. 41, 471 (2005).

[20] J. F. Ding, A. P. Zhang, L. Y. Shao, J. H. Yan, and S. He, IEEE Photon. Technol. Lett. 17, 1247 (2005).

[21] J. D. Love, W. M. Henry, W. J. Stewart, R. J. Black, S. Lacroix and F. Gonthie, Proc. Inst. Elect. Eng. J. 133, 337 (1986).

[22] R. J. Black and R. Bourbonnais, Proc. Inst. Elect. Eng. 133, 377 (1986).

[23] J. Ma, W. Tang, and W. Zhou, Appl. Opt. 35, 5206

(1996).

\section{References}

[1] S. Vasilev, and O. Medvedkov, "Long-period refractive index fiber gratings: properties, applications, and fabrication techniques," Proc. SPIE 4083, (2000).

[2] K. O. Hill, B. Malo, K. Vineberg, F. Bilodeau, D. Johnson, and I. Skinner, "Efficient mode-conversion in telecommunication fiber using externally written gratings," Electron. Lett. 26, 692 (1990). 
[3] V. Bhatia and A. M. Vengsarkar, "Optical fiber longperiod grating sensors," Opt. Lett. 21, 850 (1996).

[4] H. J. Patrick, "Self-aligning, bipolar bend transducer based on long period grating written in eccentric core fibre," Electron. Lett. 36, 1763 (2000).

[5] X. F. Chen, C. Zhang, D. J. Webb, K. Kalli, and G. D. Peng, "Highly sensitive bend sensor based on Bragg grating in eccentric core polymer fiber," IEEE Photon. Technol. Lett. 22, 850 (2010).

[6] T. Allsop, A. Gillooly, V. Mezentsev, T. EarthgrowlGould, R. Neal, D. J. Webb, and I. Bennion, "Bending and orientational characteristics of long period gratings written in D-shaped optical Fiber," IEEE Trans. Instrum.Meas. 53, 130 (2004).

[7] D. H. Zhao, X. F. Chen, K. M. Zhou, L. Zhang, I. Bennion, W. N. MacPherson, J. S. Barton, and J. D. C. Jones,"Bend sensors with direction recognition based on long-period gratings written in D-shaped fiber," Appl. Opt. 43, 5425 (2004).

[8] F. M. Araujo, L. A. Ferreira, J. L. Santos, and F. Farahi, "Temperature and strain insensitive bending measurements with D-type fibre Bragg gratings," Meas. Sci. Technol. 12, 829 (2001).

[9] Y. J. Rao, Y. P. Wang, Z. L. Ran, and T. Zhu, "Novel fiber-optic sensors based on long-period fiber gratings written by high-frequency CO2 laser pulses," J. Lightwave Technol. 21, 1320 (2003).

[10] T. Allsop, K. Kalli, K. Zhou, Y. Lai, G. Smith, M. Dubov, D. J. Webb, and I. Bennion, "Long period gratings written into a photonic crystal fibre by a femtosecond laser as directional bend sensors," Opt. Commun. 281, 5092 (2008).

[11] H. J. Patrick, C. C. Chang, and S. T. Vohra, "Longperiod fiber gratings for structure bend sensing," Electron. Lett. 34, 1773 (1998).

[12] T. Allsop, M. Dubov, A. Martinez, F. Floreani, I. Khrushchev, D. J. Webb, and I. Bennion, "Bending characteristics of fiber long-period gratings with cladding index modified by femtosecond laser," J. Lightwave Technol. 24, 3147 (2006).

[13] T. Allsop, M. Dubov, A. Martinez, F. Floreani, I. Khrushchev, D. J. Webb, and I. Bennion, "Long period grating directional bend sensor based on asymmetric index modification of cladding," Electron. Lett. 41, 59 (2005).

[14] L. Jin, W. Jin, and J. Ju, "Directional bend sensing with a CO2-laser-inscribed long period grating in a photonic crystal fiber," J. Lightwave Technol. 27, 4884 (2009).

[15] L. Y. Shao, L. Y. Xiong, C. K. Chen, A. Laronche, and J. Albert, "Directional bend sensor based on regrown tilted fiber Bragg grating," J. Lightwave Technol. 28, 2681 (2010).

[16] S. Zhang, W. G. Zhang, S. C. Gao, P. C. Geng, and X. L. Xue, "Fiber-optic bending vector sensor based on Mach-Zehnder interferometer exploiting lateral-offset and up-taper," Opt. Lett. 37, 4480 (2012).

[17] Y. Liu, L. Zhang, J. A. R. Williams, and I. Bennion, "Optical Bend Sensor Based on Measurement of Resonance Mode Splitting of Long-Period Fiber Grating," IEEE Photon. Technol. Lett. 12, 531 (2000).
[18] A. W. Synder, Optical Waveguide Theory. London, U.K: Chapman and Hall, (1983).

[19] T. Allsop F. Floreani, K. Jedrzejewski, R. Marquez, R. Romero, D. J. Webb, and I. Bennion, "Refractive index sensing with long-period grating fabricated in biconical tapered fiber," Electron. Lett. 41, 471 (2005).

[20] J. F. Ding, A. P. Zhang, L. Y. Shao, J. H. Yan, and $\mathrm{S}$. He, "Fiber-taper seeded long-period grating pair as a highly sensitive refractive-index sensor," IEEE Photon. Technol. Lett. 17, 1247 (2005).

[21] J. D. Love, W. M. Henry, W. J. Stewart, R. J. Black, S. Lacroix and F. Gonthie, "Tapered single-mode fibres and devices-Part 1: Adiabaticity criteria," Proc. Inst. Elect. Eng. J. 133, 337 (1986).

[22] R. J. Black and R. Bourbonnais, "Core-mode cutoff for finite-cladding light guides," Proc. Inst. Elect. Eng. 133, 377 (1986).

[23] J. Ma, W. Tang, and W. Zhou, "Optical-fiber sensor for simultaneous measurement of pressure and temperature: analysis of cross sensitivity," Appl. Opt. 35, 5206 (1996). 